\title{
Philippine Mental Health Act: just an act? A call to look into the bi-directionality of mental health and economy
}

Nicholle Mae Amor T. Maravilla ${ }^{1}$, Myles J oshua T. Tan ${ }^{2 *}$

${ }^{1}$ Cebu Doctors' University, Philippines, ${ }^{2}$ University of St. La Salle, Philippines

Submitted to J ournal:

Frontiers in Psychology

Specialty Section:

Health Psychology

Article type:

Opinion Article

Manuscript ID:

706483

Received on:

07 May 2021

Journal website link:

www. frontiersin.org 


\section{Conflict of interest statement}

The authors declare that the research was conducted in the absence of any commercial or financial relationships that could be construed as a potential conflict of interest

\section{Author contribution statement}

Conceived the work: MJ TT and NMATM. Drafted the article: NMATM. Critically revised the manuscript: MJ TT. Both authors read and approved the final manuscript.

\section{Keywords}

Philippines, Mental Health Act, Mental health access, mental health policy, Depression, Anxiety, Psychology, happiness, happiness \& well-being, World Happiness Index, world happiness report, culture, Europe, Nordic countries, Nordic countries of Europe, Northwestern Europe, Health and economy, Public Health, Health Policy, public health policies and politics, Mental health service, social inclusion

\section{Contribution to the field}

This article calls for an investigation into the potential bi-directionality between mental health and economics, particularly in the Philippines. It may seem intuitive to say that a healthy economy makes people happy, but little is said about the converse of this statement. Perhaps, we should look into how happy people make an economy healthy. Although we did not fully prove the existence of this bi-directional relationship, we collected evidence that points toward this as a potential truth and call on researchers to take on the challenge. 


\section{Philippine Mental Health Act:}

\section{just an act? A call to look into}

\section{the bi-directionality of mental}

\section{health and economy}

Nicholle Mae Amor Tan Maravilla', Myles Joshua Toledo Tan ${ }^{2,3^{*}}$

'College of Medicine, Cebu Doctors' University, Mandaue City, Cebu, Philippines

2Department of Natural Sciences, University of St. La Salle, Bacolod, Neg. Occ., Philippines

3Department of Chemical Engineering, University of St. La Salle, Bacolod, Neg. Occ., Philippines

* Correspondence:

Myles Joshua Toledo Tan

mj.tan@usls.edu.ph

\section{Introduction}

It may seem intuitive to say that a healthy economy makes people happy, but little is said about the converse of this statement. Perhaps, we should look into how happy people make an economy healthy. Poli et al (2019) described good mental health as "a state of well-being that allows individuals to cope with the normal stresses of life and function productively." However, the definition of good mental health may vary among cultures, values and traditions. Culture affects how people manifest symptoms, express these symptoms, deal with psychological problems, and decide whether to seek care (Eshun and Gurung, 2009). Because of these differences, the best way to enrich our understanding of mental health might be to evaluate the different perspectives of mentally healthy populations toward it (Vaillant, 2003). Further understanding of mental 
health is significant because it greatly affects the economy. According to Knapp and Wong (2020), economics has a bi-directional relationship with it. Economic decline may lead to a greater likelihood of mental-illness due to exposure to risk factors such as social exclusion, poor education, treatment costs, unemployment, and poverty. Mental health problems may also lead to a significant decline in economic activity that results from productivity losses and limited resources for treatment.

\section{The State of Mental Health in the Philippines}

The Philippines is an archipelago of over 7000 islands, with over 120 languages, and numerous religions (Lally et al, 2019). The culture, beliefs, and perceptions of Filipinos vary in almost every respect. There is also a popular belief that depression and anxiety are non-existent, and that mental illnesses are something to be ashamed of. A qualitative study conducted by Tanaka et al (2018) showed that this stigma is considered to be an effect of the public belief about mental disorders which consist of three themes: First is familial problems, wherein the family rejects or disowns the family member who suffers from a mental disorder because they believe that it can be inherited. Second is unrealistic pessimism and optimism about the severity of the disorder, wherein the mentally ill either would certainly suffer from severe functional impairment or would be able to overcome any psychological suffering by themselves. Last is the oversimplified chronic course, wherein people without mental illnesses apply an acute illness model to those ill, and expect full recovery in the short term.

Because of this stigma, mental health has been given very little attention by the Philippine government and public sectors. Even after the country has recently passed its first Mental Health Act and Universal Health Care Law, only $5 \%$ of the healthcare expenditure is directed toward mental health. Also, there are only 7.76 hospital beds and 0.41 psychiatrists per 100,000 people (Department of Health [DOH], 2018; WHO-AIMS, 2007). This ratio 
was known to be lower than other Western Pacific countries with similar economic statuses, like Malaysia and Indonesia (Lally et al, 2019). The Philippine government does not even provide economic support for organizations that have been involved in the formulation and implementation of mental health policies and legislation (WHO-AIMS, 2007). Consequently, mental illness has become the third most common disability in the Philippines, wherein six million Filipinos live with depression and anxiety. Because of this, the country has the third highest rate of mental disorders in the Western Pacific (Martinez et al, 2020). Also, the Philippine WHO Special Initiative for Mental Health conducted in 2020 showed that $\geqslant 3.6$ million Filipinos suffer from at least one kind of mental, neurological, or substance use disorder.

Filipinos are generally unhappy not only because of poor economic conditions (unemployment, low salary, etc.), but also because of pressures arising from high expectations from family and society. According to Palaganas et al (2017), even the most educated Filipinos like physicians and nurses migrate abroad where they are given more respect and quality of practice, which greatly develop their wellbeing. This, however, is contrary to Martinez et al who argued that although Filipinos enjoy more opportunities and higher wages abroad, mental health issues still exist among Overseas Filipino Workers due to language barriers, immigration status, lack of insurance, and discrimination. 


\section{THE STATE OF MENTAL HEALTH IN THE PHILIPPINES}

THE MENTAL HEALTH ACT \& UNIVERSAL HEALTH CARE LAW

was established to enhance the delivery of mental health services and to promote and protect the rights of the Filipinos utilizing psychiatric, neurologic, and psychosocial health services. However

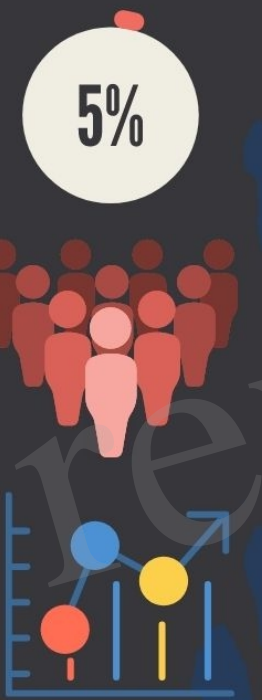

Only $5 \%$ of the healthcare expenditure is directed toward mental health services. least one kind of mental, neurological, or substance use disorder rate of mental disorders in the Wester Pacific Region.

\section{STIGMA AND DISCRIMINATION}

toward mental issues became part of the

Filipino culture, and this has greatly affected the people and the economy.

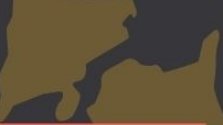

(1)
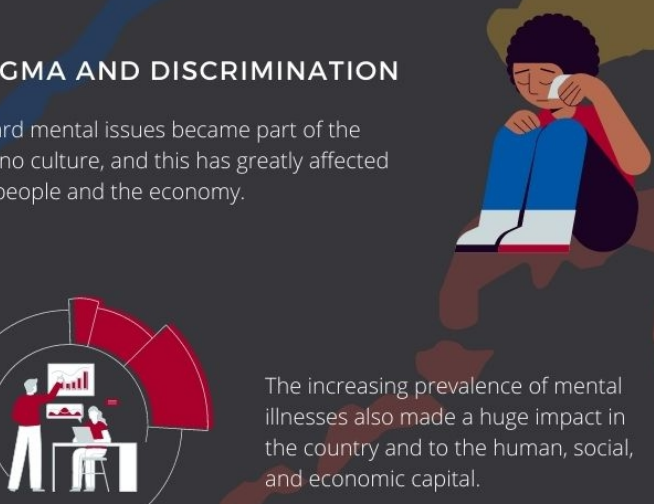

The increasing prevalence of mental illnesses also made a huge impact in the country and to the human, social, and economic capital.

The Philippines is a developing country that struggles to obtain economic stability because of outdated ways that result in gaps in mental health promotion, which in turn bleed into the economy.

Figure 1. An infographic that summarizes the state of mental health in the Philippines. 


\section{A Happy Northwestern Europe}

Mental health is associated with either happiness or unhappiness, and is significant to Nordic life. A good reason why Nordics are happy is their system that provides security, free education, and a reasonable balance of work and personal time (In The Shadow of Happiness, 2018). Humanitarianism is the root of their culture that has led to economic growth and reconversion of cities. Innovation and development were also attributed to their kindness toward the multicultural neighborhoods of their country (Culture, Cities, and Identity in Europe, 2016). Aside from culture, the government also plays an important role in developing these countries. Northwestern European countries have among the highest scores in the World Governance Indicator. This is because of the digital tools that they use to optimize management, service delivery, and overall state capacity. National websites are established to allow citizens' participation in policymaking and to provide feedback on public services. Thus, the collaboration between governments and their citizens is able to strengthen research, monitoring, and the evaluation framework of policymaking. It also promotes transparency and trust between them. In addition, health has always been their top priority. Therefore, public services such as healthcare and disability services are supported by the government (World Bank, 2021).

\section{The Philippines and Northwestern Europe - A Comparison}

The World Bank (2021) has emphasized that mental health is part of the strategy to improve disadvantaged economies. In order to achieve this, people should be in positive mental and emotional states of enjoyment and contentment, id est "happiness" (Richards et al, 2015). However, according to Hart et al (2018) and Wahlbeck (2015), socioeconomic factors like poverty, poor education, unemployment, and high debt, which are mostly experienced by Filipinos, affect happiness. 
In order to promote happiness, more job opportunities and higher wages should be provided since low-wage earners are more prone to psychological distress (Kronenberg et al, 2017), while higher wages protect individuals against dissatisfaction and unhappiness (In The Shadow of Happiness, 2018). Palaganas et al (2017) showed that the decision of the workers to migrate is mainly influenced by their job dissatisfaction with income, workload, and infrastructure. However, increasing the wages of low-wage earners would not entirely enhance their mental health (Kronenberg et al, 2017). Thus, mental health promotion should still be implemented.

Being that mental health issues have been widely neglected in the Philippines and migration has resulted in the loss of investments in human capital (Palaganas, 2017), it would be best to take steps to attempt to adopt the mental health promotion strategy of Europe as nine of the 10 happiest countries in the world - Finland, Denmark, Switzerland, Iceland, Netherlands, Norway, Sweden, Germany, and Austria are in Northwestern Europe (World Happiness Report, 2021). This is perhaps because of their good economic status and quality healthcare. Mental health promotion in these countries proved to be more effective because it was introduced into the political agenda using a different approach. Neither the prevalence of mental illness nor the need for resources was emphasized. Instead, mental health was highlighted as a fundamental component of public health, and as having a significant effect on individual countries and their human, social, and economic capital (Wahlbeck, 2015). If the $\mathrm{DOH}$ in the Philippines would use this approach, the government would view the bottomline of the economic crisis in a different light. Realizing this would provide a new perspective to managing the economy.

However, the Filipino people distrust the government. This leads to a disunity between the two groups. This is contrary to the experiences in Northwestern Europe, where individuals trust and socially interact with their neighborhoods. Finland, for instance, which ranked highest on 
measures of mutual trust, enjoys the sentiment that their lives and livelihoods are protected even during times of the pandemic (World Happiness Report, 2021 ; Hart et al, 2018). The architects of the mental health program of the WHO in Europe, its member states, and their partners worked together to develop and implement mental health policies and legislations that reflect the vision of the WHO that there is "no health without mental health" (WHO European Region, 2020). If the Philippines were also to endeavor toward this sense of solidarity, there, too, would be improvements in the healthcare system that would send ripples throughout the economy.

Mental health is not well-established in the Philippines because of the dearth of investments channeled toward research. Thus, Filipino mental health workers cannot fully utilize their skills due to outdated practice guidelines and inappropriate curricula (Palaganas et al, 2017) unlike in Europe where guidance on economic crises and mental health are based on carefully reviewed research (Carrasco et al, 2016). The implementation of community mental health services are also based on empirical clinical evidence. Through this, they are able to recognize gaps that exist between the needs of the population and actual service provision (Semrau, 2011). This commitment has led to technological innovations such as e-Mental Health after evaluating the efficacy of delivering mental health services (Gaebel et al, 2020).

\section{Discussion}

Thus far, the Philippine Mental Health Act has been nothing more than "just an act." Nonetheless, there is still hope that the provision of mental healthcare will be recognized as a significant need to ameliorate life and economy. However, gradual change should begin with norms ingrained in culture before governmental reforms could be enjoyed, as these, too, are 
products of social norms themselves. Moreover, social stigma and discrimination are the toxic traits that misshape Filipino culture.

This article is a call for Filipinos to view mental health issues in a different light and to impel government and public sectors to prioritize them and to set the Philippine Mental Health Act into motion. Depression and anxiety should be recognized as disorders, not mere illusions. Families must be listeners and comforters of the mentally ill, not constigators. Filipinos must also understand that there is a complex process in managing mental health issues and full recovery could not be achieved over a short period of time.

Furthermore, this is the time to drop the romanticization of Filipino resiliency because the truth is that the Filipino is not genuinely happy. A picture of a smiling Filipino does not equate to a happy Filipino because the mentally ill know how to smile too. The Philippines is a developing country that struggles to obtain economic stability because of outdated ways that result in gaps in mental health promotion, which in turn, bleed into the economy. The increasing prevalence of mental illnesses bears great impact on human, social, and economic capital. This may be true not only in the Philippines, but also in other developing countries. Hence, future researchers should look into verifying the converse of the seemingly intuitive idea that a healthy economy makes people happy. Happy people will make our economy healthy! 


\section{References}

Andreasson, U. and Birkjaer, M. (2018). In the Shadow of Happiness. Nordic Council of Ministers. http://dx.doi.org/10.6027/ANP2018-799

Carrasco, M., Lacko, S., Dom, G., Christodoulou, N., Samochowiec, J. et al (2016). EPA guidance on mental health and economic crises in Europe. Eur Arch Psychiatry Clin Neurosci 266: 89124. doi: 10.1007/s00406-016-0681-x.

Department of Health (2018). Mental Health Program. https://doh.gov.ph/national-mentalhealth-program.

European Economic and Social Committee (2016). Culture, Cities, and Identity of Europe. https://www.eesc.europa.eu/resources/docs/qe-01-16-463-en-n.pdf.

Gaebel, W., Lukies, R., Kerst, A., Stricker, J., Diekmann, S. et al (2020). Upscaling e-mental health in Europe: a six-country qualitative analysis and policy recommendations from the eMEN project. European Archives of Psychiatry and Clinical Neuroscience. https://doi.org/10.1007/s00406-020-01133-y.

Hart, E., Lakerveld, J., Mckee, M., Oppert, J., Rutter, H. et al (2018). Contextual correlates of happiness in European adults. PLOS ONE 13(1). https://doi.org/10.1371/journal.pone.0190387.

Knapp, M. and Wong, G. (2020). Economics and mental health: the current scenario. World Psychiatry 19 (1):3-14. doi: 10.1002/wps.20692.

Kronenberg, C., Jacobs, R., and Zucchelli, E. (2017). The impact of the UK National Minimum Wage on mental health. SSM - Population Health 3: 749-

755. https://doi.org/10.1016/j.ssmph.2017.08.007.

Lally, J., Tully, J., \& Samaniego, R. (2019). Mental health services in the Philippines. BJPsych International 16(3): 62-64. doi:10.1192/bji.2018.34.

Martinez, A., Co, M., Lau, J., Brown, J. (2020). Filipino help-seeking for mental health problems and associated barriers and facilitators: a systematic review. Social Psychiatry and Psychiatric Epidemiology 55:1397-1413 https://doi.org/10.1007/s00127-020-01937-2.

Palaganas, E., Spitzer, D., Kabamalan, M., Sanchez, M., Caricativo, R. et al (2017). An examination of the causes, consequences, and policy responses to the migration of highly trained health personnel from the Philippines: the high cost of living/leaving - a mixed method study. Human Resource for Health 15:25. doi: 10.1186/s12960-017-0198-z.

Poli, P., Pablo, G. Micheli, A., Nieman, D., Corell, C. et al (2020). What is a good mental health: A scoping review. European Neuropsychopharmacology 31:33-

46. https://doi.org/10.1016/j.euroneuro.2019.12.105.

Richards, J., Jiang, X., Kelly, P., Chau, J., Bauman, A., and Ding, D. (2015). Don't worry, be happy: cross-sectional associations between physical activity and happiness in 15 European countries. BMC Public Health 15:53. doi: 10.1186/s12889-015-1391-4.

Semrau, M., Barley, E., Law, A., and Thornicoft, G. (2011). Lessons learned in developing community mental health care in Europe. World Psychiatry 10 (3): 217-225. doi: 10.1002/j.20515545.2011.tbooo6o.x. 
Tanaka, C., Tuliao, M., Tanaka, E., Yamashita, T., and Matsuo, H. (2018). A qualitative study on the stigma experienced by people with mental health problems and epilepsy in the Philippines. BMC Psychiatry 18:325. https://doi.org/10.1186/s12888-018-1902-9. Vaillant, G. (2003). Mental Health. American Journal of Psychiatry 160 (8): 1373 1384. https://doi.org/10.1176/appi.ajp.160.8.1373.

Wahlbeck, K. (2015). Public mental health: the time is ripe for translation of evidence into practice. World Psychiatry 14(1): 36-42. doi: 10.1002/wps.20178.

World Bank Group (2021). Data, Digitalization, and Governance. doi: 10.1596/978-1-46481698-7.

World Happiness Report (2021). In a Lamentable Year, Finland Again is the Happiest Country in the World. https://worldhappiness.report/blog/in-a-lamentable-year-finland-again-is-thehappiest-country-in-the-world/.

World Health Organization (2018). Mental health: strengthening our response. https://www.who.int/news-room/fact-sheets/detail/mental-health-strengthening-ourresponse.

World Health Organization - Assessment Instrument for Mental Health Systems (2007). WHOAIMS Report on Mental Health Systems in the Philippines. https://www.who.int/mental_health/evidence/philippines_who_aims_report.pdf.

World Health Organization - European Region (2018). Mental

Health. https://www.euro.who.int/en/health-topics/noncommunicable-diseases/mentalhealth/mental-health. 


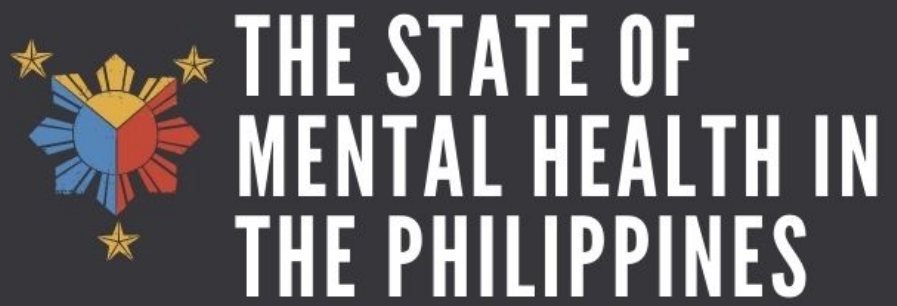

THE MENTAL HEALTH ACT \& UNIVERSAL HEALTH CARE LAW

was established to enhance the delivery of mental health services and to promote and protect the rights of the Filipinos utilizing psychiatric, neurologic, and psychosocial health services. However
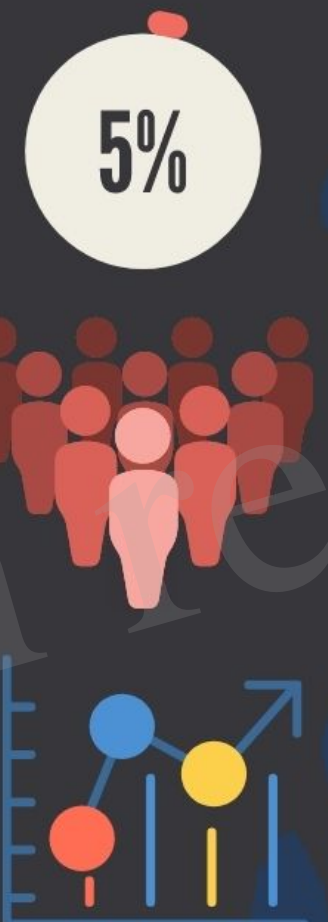

Only $5 \%$ of the healthcare expenditure is directed toward mental health services.

$\geqslant 3.6$ million Filipinos suffer from at least one kind of mental, neurological, or substance use disorder

The Philippines has the third highest rate of mental disorders in the Western Pacific Region.

\section{STIGMA AND DISCRIMINATION}

toward mental issues became part of the Filipino culture, and this has greatly affected the people and the economy.
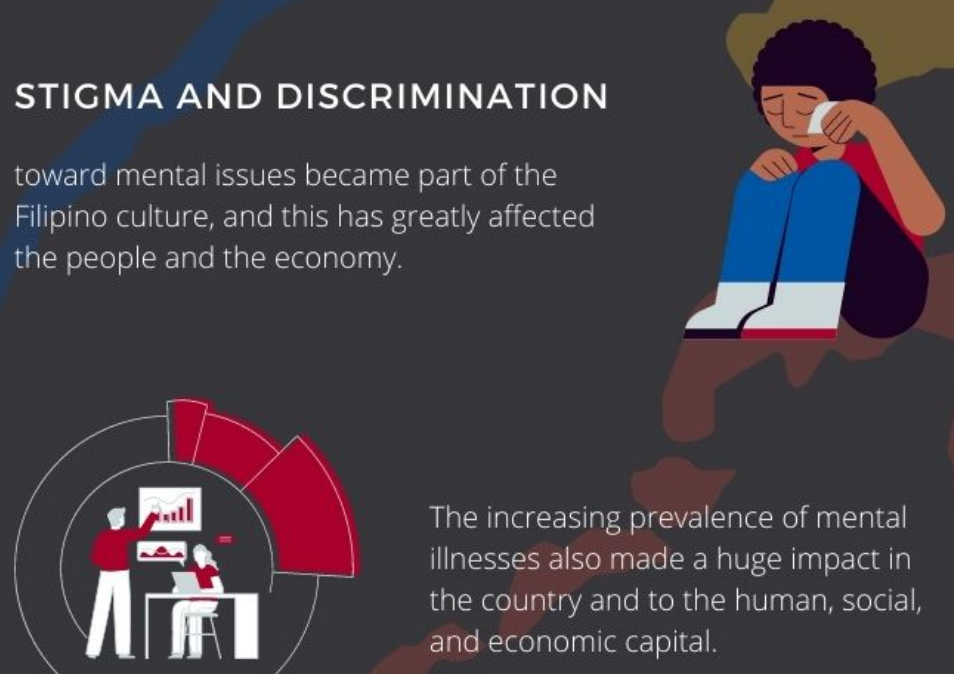

The increasing prevalence of mental illnesses also made a huge impact in the country and to the human, social, and economic capital. 